\title{
Minimum Variance Algorithm-Based Correlation Analysis between Body Mass Index and the Malignant Degree of Prostate Cancer Mediated under Ultrasound Images
}

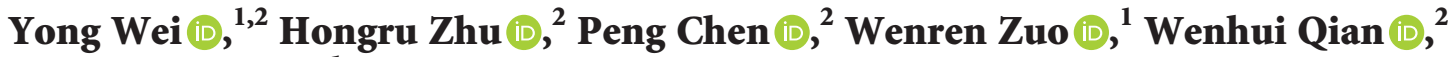 \\ and Qingyi Zhu (iD) \\ ${ }^{1}$ Department of Urology, Affiliated Hospital of Nanjing University of Chinese Medicine, 155 Hanzhong Road, \\ Nanjing 210029, China \\ ${ }^{2}$ Department of Urology, Nanjing Gaochun People's Hospital, Nanjing 211300, China
}

Correspondence should be addressed to Qingyi Zhu; 20193061@njucm.edu.cn

Received 22 October 2021; Revised 1 December 2021; Accepted 3 December 2021; Published 23 December 2021

Academic Editor: M Pallikonda Rajasekaran

Copyright (c) 2021 Yong Wei et al. This is an open access article distributed under the Creative Commons Attribution License, which permits unrestricted use, distribution, and reproduction in any medium, provided the original work is properly cited.

This study was to explore the correlation between the malignant degree of prostate cancer (PCa) and body mass index (BMI) mediated by ultrasound images under multioperator algorithm (MOA) based on minimum variance (MV) algorithm. MOA was established by optimizing the smoothing technique and diagonal loading algorithms of MV, and its quality and processing speed of ultrasound images were compared with other algorithms. Ninety two patients were selected as the subjects investigated, who had transrectal prostate biopsy mediated by ultrasound to be diagnosed as PCa in the hospital. Based on Gleason score and prostate specific antigen (PSA) value, all patients were divided into a high-risk PCa group (a high-risk group) and a non-high-risk PCa group (a non-high-risk group). The proportion of obese patients in the two groups was compared. The logistic regression analysis method was applied to analyze related factors of PCa development, and Pearson correlation was for analyzing the correlation between Gleason score and BMI of patients. The results showed that the number of patients in the high-risk group was greater than that of the non-high-risk group $(P<0.05)$, while the proportion of nonobese patients in the non-high-risk group was markedly higher than that of the higher-risk group $(P<0.01)$. Both PSA and BMI were obviously correlated with the development of high-risk PCa $(P<0.05)$, and there was an extreme positive correlation between BMI and Gleason score $(r=0.661$ and $P=0.007)$. It indicated that MOA was established based on conventional MV, which could increase the ultrasonic image quality and calculation speed. Besides, BMI was a risk factor of high-risk PCa and was positively correlated with malignant degree of PCa, which provided a referable evidence for clinical evaluation of malignant degree of PCa.

\section{Introduction}

PCa is a common malignant tumor of male reproductive system, and its incidence ranks the second among male malignant tumors [1], which has seriously threatened men's healthy life at present. Nowadays, the incidence of PCa in developed countries such as some European countries and America is obviously higher than that of China. However, with the improvement of life quality in recent years, the prevalence and diagnosis rates of PCa in China showed an obvious rising trend [2]. Current studies on factors related to malignant degree of $\mathrm{PCa}$ found that risk factors for malignant degree of PCa mainly include age, race, and inheritance.
However, the influences of dietary habits, lifestyle, hormones, inflammation, and obesity on PCa have not been confirmed. Some studies have shown that changes in BMI and blood lipid level are correlated with the emerging of PCa to a certain extent [3]. In addition, some studies have found that the mortality rate of patients with PCa in countries with high dietary fat intake is higher than the rate of countries with low dietary fat intake, indicating that excessive fat intake has a certain influence on the development of cancer [4]. However, the influence factors for malignant degree of PCa are not clear at present.

Ultrasonic imaging is a commonly applied diagnostic technique in clinical medicine and has become a common 
method for detection of many diseases because of its simple operation, noninvasiveness, and wide application range. However, the commonly applied delay-and-sum (DAS) algorithm has poor imaging quality [5]. Although DAS is optimized by a large number of high-definition imaging algorithms, there are some disadvantages such as high computational complexity and difficulty in real-time image acquisition [6]. MV adaptive beam former algorithm can not only improve the imaging performance of the ultrasound imaging system but also effectively suppress nonaxial signals, so as to obtain higher-definition images [7]. Although MV can meet the needs of most medical imaging, it still cannot provide detailed imaging for some complex diagnosis.

Based on the influence of conventional MV on imaging performance, multiple improved operators were introduced to establish a new ultrasound imaging algorithm. Ninety two patients were collected as the subjects investigated, who had transrectal prostate biopsy mediated by ultrasound to be diagnosed as PCa in the hospital from June 2018 to March 2020, to explore the correlation between BMI and malignant degree of $\mathrm{PCa}$, so as to provide a reference for clinical evaluation of malignant degree of PCa.

\section{Materials and Methods}

2.1. The Subjects Investigated and Grouping. Ninety two patients, who were diagnosed with PCa through transrectal prostate biopsy mediated by ultrasound in hospital from June 2018 to March 2020, were collected as the subjects investigated. The criteria for inclusion were defined to include patients who were diagnosed as PCa, had no surgical history of PCa before undergoing transrectal prostate biopsy, and had no radiotherapy and chemotherapy before taking transrectal prostate biopsy. The criteria for inclusion were defined to include patients suffering from $\mathrm{PCa}$ combined with other malignant tumors and had severe hepatic and renal dysfunction. Based on whether a patient's BMI value was greater than $25 \mathrm{~kg} / \mathrm{m}^{2}$, the subjects investigated were divided into obese and nonobese patients. In addition, the subjects investigated were grouped into a high-risk group and a non-high-risk group based on Gleason scores of patients with PCa who were over or equaled to 8 points, and the PSA value was greater than or equaled to 20. The process had been approved by Ethics Committee of hospital, and all patients included in the study had signed informed consents.

\subsection{The Flow of Multioperator Ultrasound Imaging Algorithm.} Multioperator ultrasound imaging algorithm mainly included three parts (signal delay, weight calculation, and signal superposition). As shown in Figure 1, the weight calculation module was mainly optimized on the basis of ultrasound imaging algorithm through the addition of forward and backward spatial smoothing operator (FB), diagonal loading operator (DL), and eigenspace-based operator (ESB), so as to improve the quality of ultrasound images (the improvement was highlighted as yellow in Figure 1).
2.3. Multioperator Ultrasound Imaging Algorithm. The MV beamforming algorithm was a subaperture composed of $M$ continuous input channels, which were separated by a receiving array composed of $N$ continuous input data channels. Besides, the received data were $N-M+1$, and the corresponding noise covariance matrix was calculated as follows:

$$
R_{M}=\frac{1}{N-M+1} \sum_{i=0}^{N-M} x_{i} x_{i}^{A},
$$

where $x_{i}$ is the vector of $M \times 1$, which was composed of signal data in the $i^{\text {th }}$ group of subaperture, and it could be calculated as the following equation:

$$
x_{i}=\left[s_{i}, s_{i+1}, \cdots, s_{i+M-1}\right]^{t} .
$$

The forward and backward spatial smoothing technology had a good effect on the improvement of estimation accuracy of noise covariance matrix [8], and its algorithm was $R_{B}=K \cdot R_{M} \cdot K$ ( $K$ was the exchange matrix). Therefore, a more accurate estimation algorithm could be obtained through forward and backward spatial smoothing:

$$
R_{F}=\frac{1}{2}\left(R_{M}+R_{B}\right)
$$

The diagonal loading method was often applied to promote the robustness of beamforming algorithm [9], and its specific calculation is as follows:

$$
R_{D}=R_{M}+\beta \cdot \operatorname{trace}\left(R_{M}\right) \cdot B,
$$

where $\beta$, trace, and $B$ stood for diagonal loading capacity, trace of a matrix, and unit matrix, respectively. The diagonal loading capacity should not be too large [10] and should meet the condition $\beta \ll 1 / M$, and the usual data range was $\beta \in[1 / 10 M],[1 / 100 M$,$] . The signal characteristic spatial$ distribution was employed to optimize the ultrasound imaging quality, and the covariance matrix of MV was expressed as the following equation:

$$
R_{M}=W \wedge W^{A}=W_{R} \wedge W_{R}^{A}+W_{H} \wedge W_{H}^{A},
$$

where $\wedge=\operatorname{diag}\left(\eta_{1}, \eta_{2}, \cdots \eta_{M}\right), \quad \eta_{1} \geq \eta_{2} \geq \cdots \geq \eta_{M}$, and $\eta$ represented eigenvalue of covariance matrix. $W=\left[\omega_{1}, \omega_{2} \cdots \omega_{M}\right]$, where $\omega_{i}$ was the eigenvector corresponded to $\eta_{\mathrm{i}}$. $W_{R}$ expressed signal subspace, and it could be calculated by $W_{R}=\left[\omega_{1}, \omega_{2}, \cdots \omega_{\mathrm{Hsig}}\right]$ (Hsig stood for the number of large eigenvalue). Thus, the optimized weight vector could be expressed as $U_{E}=\mathrm{W}_{R} \mathrm{~W}_{R}^{A} U_{M}$, and $U_{M}$ stood for the weight vector obtained by MV.

The signal coherence was measured by the phase information of ultrasound echo data to obtain a coherent factor value, so as to avoid the focusing error caused by signal coherence [11]. Besides, coherence factor was expressed as follows:

$$
C_{s}=\left|1-\sqrt{1-\left(\frac{1}{N} \sum_{i=0}^{N-1} p_{i}\right)^{2}}\right|^{q},
$$



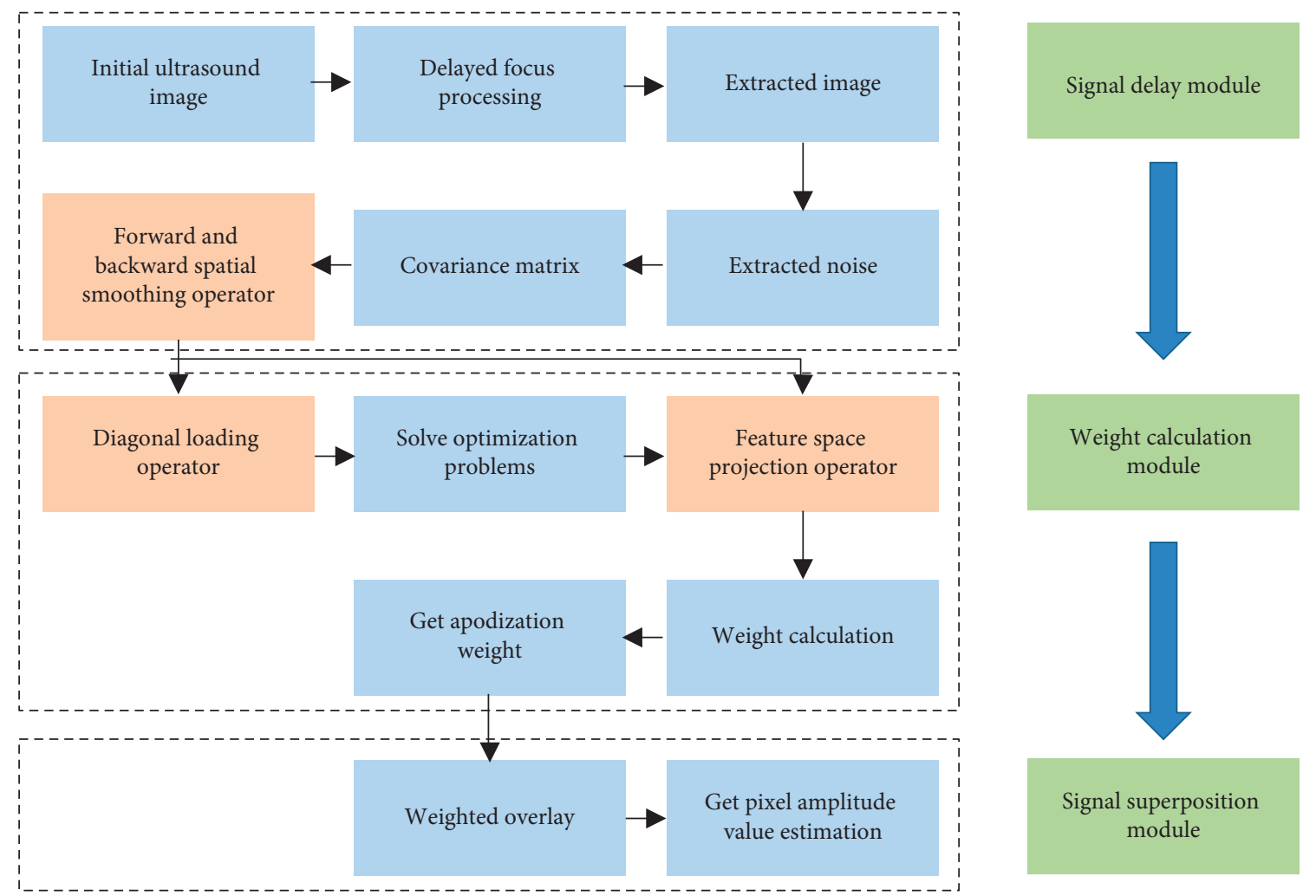

FIGURE 1: Basic flow and optimization of multioperator ultrasound imaging algorithm.

where the value of $q$ was applied to regulate the sensitivity of the coherent factor, which met the condition $q \geq 0$ and $p_{i}$ represented the value of sample points, which satisfied the condition $p_{i}=\left\{\begin{array}{ll}+1, & s_{i} \geq 0 \\ -1, & s_{i}<0\end{array}\right.$.

2.4. The Criteria for Gleason Score. Gleason score was a commonly applied pathological classification method for prostate cancer. The higher the Gleason score of a patient, the worse the malignant degree of PCa. Gleason score was over or equaled to 8 points and PSA value was greater than or equaled to 20, which meant a patient suffered from highrisk PCa. Gleason score equaled to 7 points and PSA value ranged from 10 to 20 , which showed a patient had intermediate-risk PCa. Gleason score was less than or equaled to 6 points and the PSA value was less than 10, indicating that a patient suffered from low-risk PCa [12].

2.5. Statistical Methods. SPSS20.0 statistical software was adopted to process the experimental data. The normal distribution test was for measurement data that were expressed as mean \pm standard deviation $(\bar{x} \pm s)$, and $t$-test was for comparison between the two groups. The count data were represented by a percentage (\%) and examined by the $\chi^{2}$ test. In addition, the logistic regression analysis method was applied to analyze the risk factors of PCa and high-risk PCa. $P<0.05$ expressed the difference was statistically significant.

\section{Results}

3.1. Analysis on the Imaging Quality of Multioperator Ultrasonic Imaging Algorithm. The horizontal resolution of optimized MOA was compared with conventional MV, FB, $\mathrm{DL}, \mathrm{ESB}$, symbolic coherence factor (SCF), and generalized coherence factor (GCF), and the results are shown in Figure 2. As the ratio of the sliding window length/the number of elements $(\mathrm{M} / \mathrm{N})$ increased, the horizontal resolution of MOA was in a stable state. The MV and FB algorithm showed larger fluctuations with the increase of the $M / N$ value. Meanwhile, Figure 3 indicated the imaging contrast under these seven algorithms was analyzed, and the optimized MOA in the study was least affected by the $M / N$ value.

3.2. Analysis on the Imaging Speed of Multioperator Ultrasound Imaging Algorithm. Figure 4 shows that the imaging frame rates of different algorithms were analyzed, and MOA in this study had a greatly smaller fluctuation range than other algorithms as the $M / N$ value increased. The speed-up ratios of different algorithms were compared and analyzed (Figure 5), all algorithms achieved the speed-up ratio of more than a thousand times, while the speed-up ratio of MOA remained a stable trend with the $M / N$ value increasing, and change of its fluctuation was obviously smaller than the others.

3.3. Comparison on the Clinical Data of Patients in the Two Groups. The age, weight, height, BMI, PSA, and other basic data of the patients in two groups were compared and 


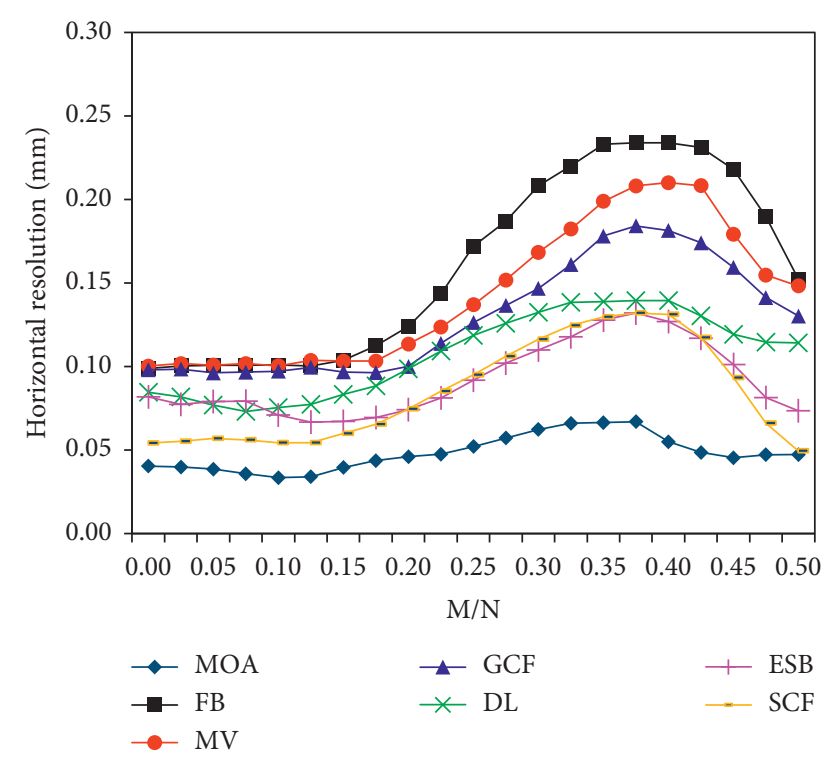

FIgURE 2: Comparison on the ultrasound imaging resolution ratio of different algorithms.

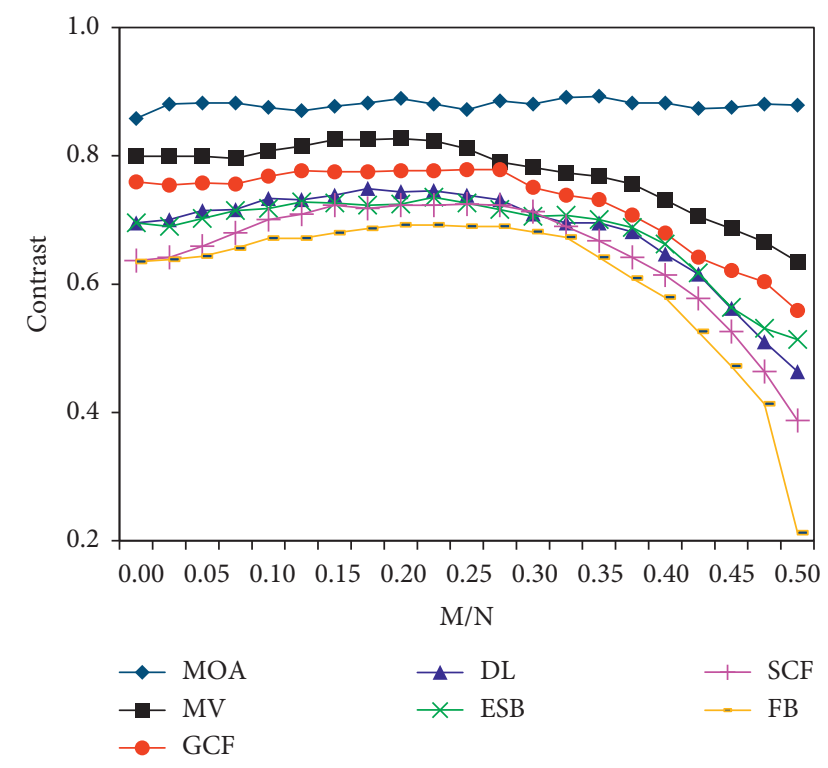

FIgURE 3: Comparison on the ultrasound imaging contrast of different algorithms.

analyzed, as shown in Table 1. Therefore, there was no substantial difference in age, height, and weight among the patients in the two groups $(P>0.05)$; BMI and PSA of patients in the non-high-risk group were lower than those of the high-risk group, showing quite considerable differences among the patients in the two groups $(P<0.01)$.

3.4. Results of Ultrasound Examination in Patients with Prostate Cancer. Patients with PCa underwent the transrectal prostate biopsy mediated by ultrasound, and the ultrasound examination results are shown in Figure 6. Furthermore, Figures 6(a) and 6(b) show the images of a

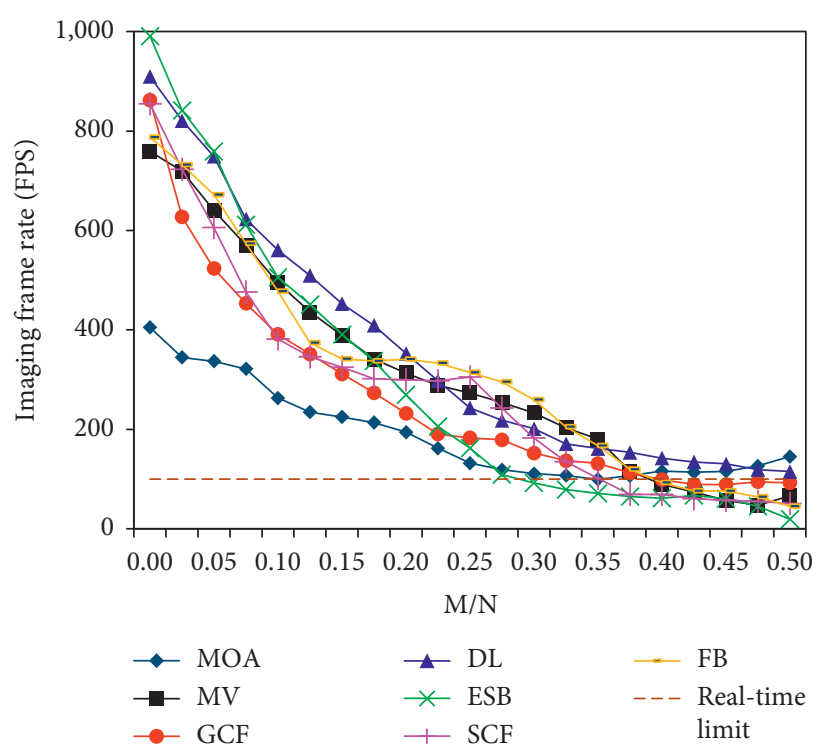

FIgURE 4: Comparison on the ultrasound imaging frame rate of different algorithms.

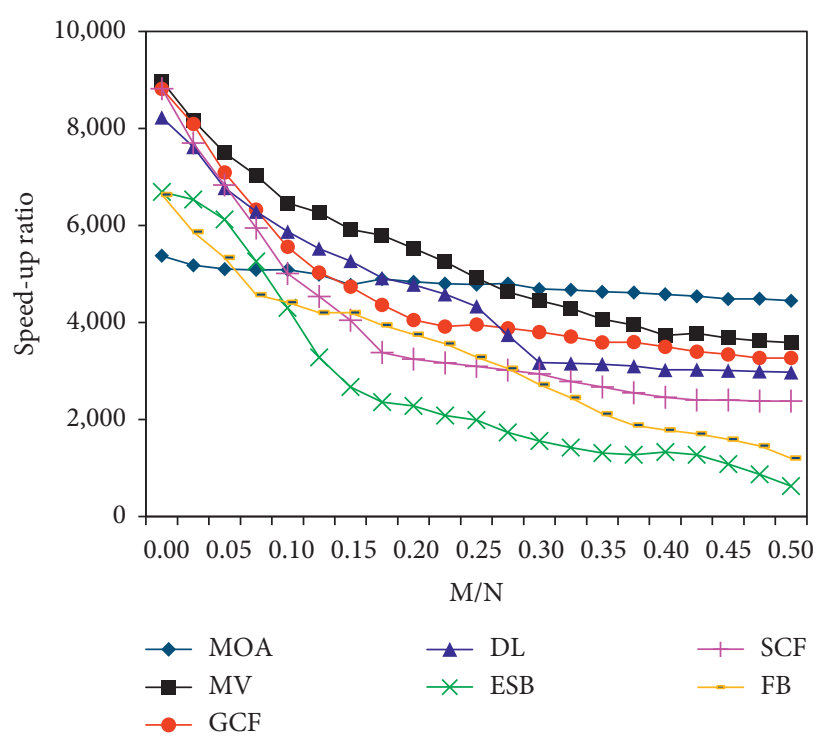

FIGURE 5: Comparison on the ultrasound imaging speed-up ratios of different algorithms.

healthy person's prostate. The ultrasound image of a patient with PCa illustrated that there was no obvious difference in the internal and external glands, the shape was irregular (Figure 6(c)), blood supply was abundant, blood flow signal was enhanced (Figure 6(d)), the continuity of bladder and rectal wall was interrupted, distant metastasis happened (Figure 6(e)), the capsule was not continuous, and internal echo was uneven (Figure 6(f)).

3.5. Analysis on the Obese Proportion of Patients with Prostate Cancer. The obesity ratio of all patients was analyzed. Figure 7 (a) indicated that obese patients with PSA accounted for $78.26 \%$, while the proportion of nonobese PSA patients 
TABLE 1: Comparison on the basic data of patients in the two groups.

\begin{tabular}{|c|c|c|c|c|}
\hline Factors & Non-high-risk group $(n=47)$ & High-risk group $(n=45)$ & $t$ or $\chi^{2}$ value & $P$ value \\
\hline Age (years old) & $68.73 \pm 1.88$ & $70.16 \pm 2.57$ & 0.268 & 0.653 \\
\hline Weight $(\mathrm{kg})$ & $67.98 \pm 9.21$ & $69.13 \pm 7.54$ & -0.793 & 0.263 \\
\hline Height (cm) & $166.36 \pm 12.14$ & $167.15 \pm 11.89$ & 0.403 & 0.335 \\
\hline BMI $\left(\mathrm{kg} / \mathrm{m}^{2}\right)$ & $25.13 \pm 1.98$ & $27.52 \pm 2.19$ & 0.059 & $0.007^{* *}$ \\
\hline PSA (ng/mL) & $31.85 \pm 9.94$ & $97.26 \pm 16.92$ & 0.073 & $0.002^{* *}$ \\
\hline
\end{tabular}

** expressed there was a very marked difference $(P<0.01)$ in contrast to the non-high-risk group.

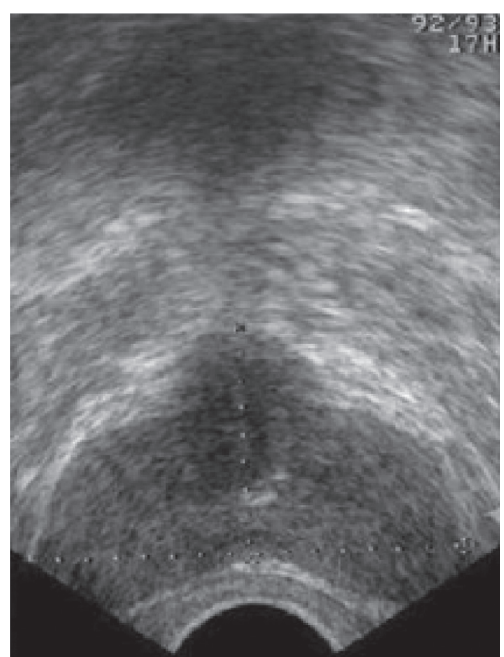

(a)

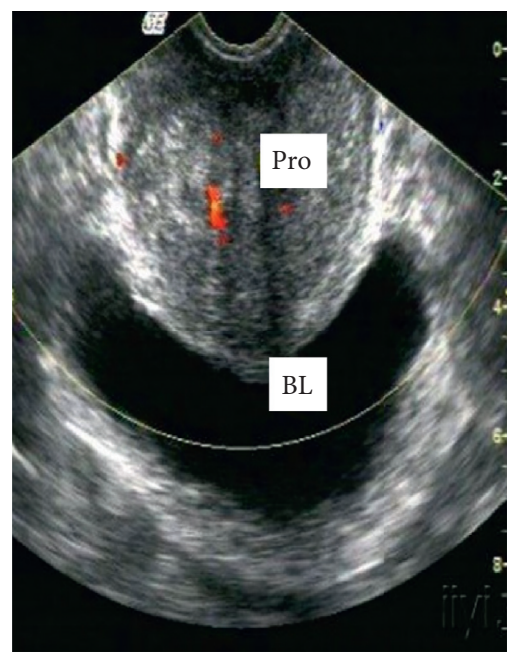

(d)

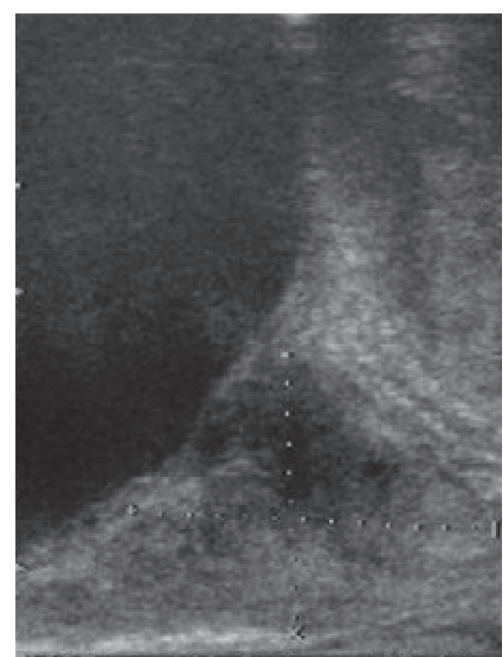

(b)

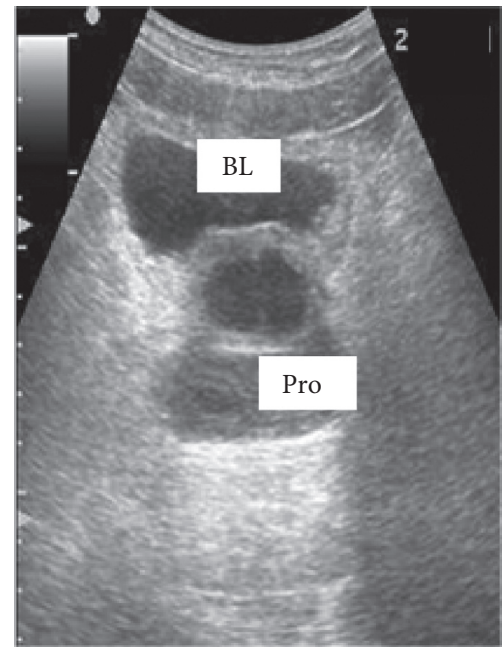

(e)

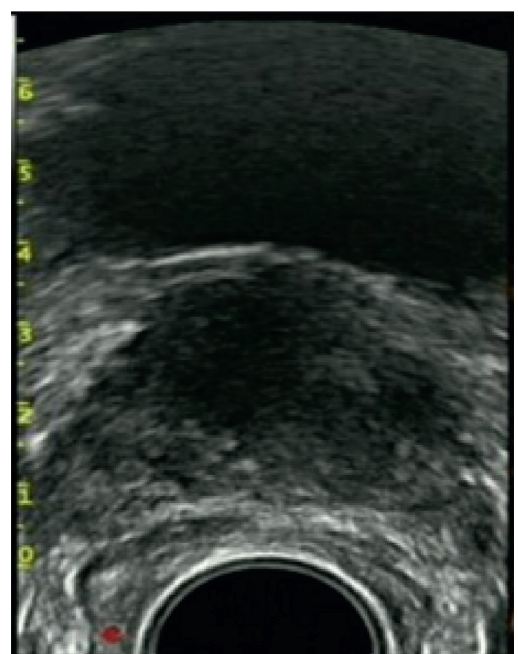

(c)

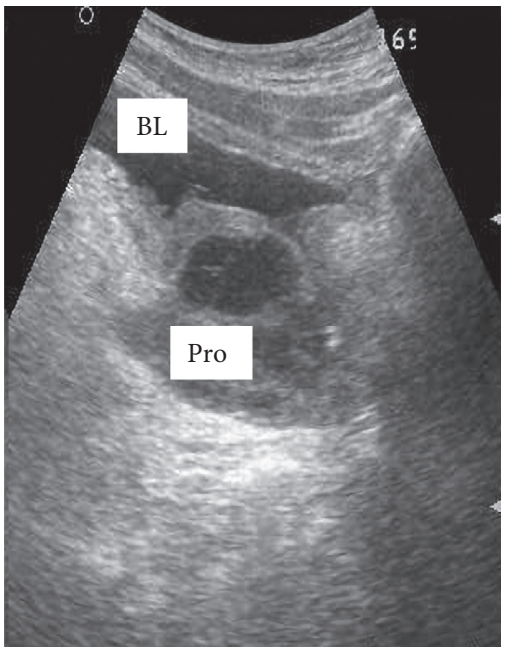

(f)

Figure 6: Images of PCa ultrasound examination. (a) and (b) The ultrasound images of a healthy person's transrectal prostate transection and vertical section, respectively. (c) The ultrasound image of a 60-year-old patient who was diagnosed as PSA with $63 \mathrm{ng} / \mathrm{mL}$ PSA. (d) The ultrasound image of a 56-year-old patient who was diagnosed as PSA. (e) The ultrasound image of a 64-year-old patient who was diagnosed as PSA. (f) The ultrasound image of a 43 -year-old patient who was diagnosed as PSA.

was only $21.74 \%$. Therefore, the proportion of obese patients with PSA was obviously greater than that of nonobese patients, with a statistically significant difference $(P<0.05)$. There was a further analysis on the proportion of obese patients in the two groups. Figure 7 (b) demonstrated that the proportions of obese patients in the high-risk and nonhigh-risk group were $58.33 \%$ and $41.67 \%$, respectively. Besides, the proportion of obese patients in the high-risk group was extremely higher than the proportion of the nonhigh-risk group $(P<0.05)$. On the contrary, the proportion 


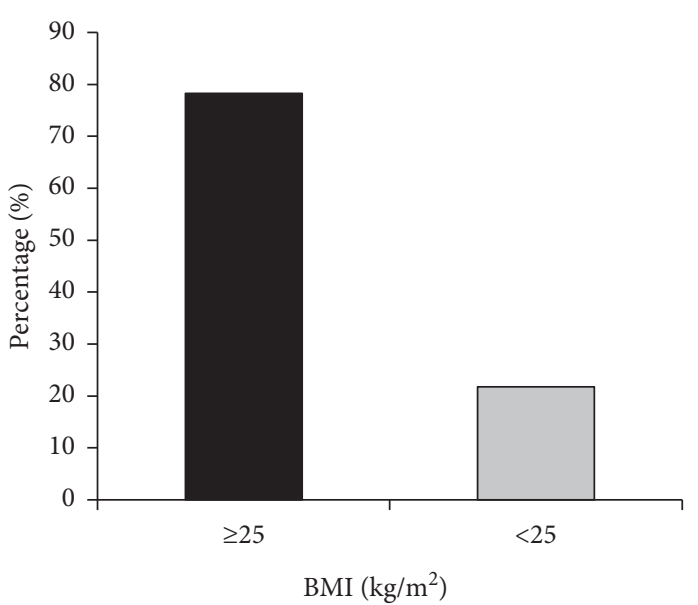

High-risk group

Non-high-risk group

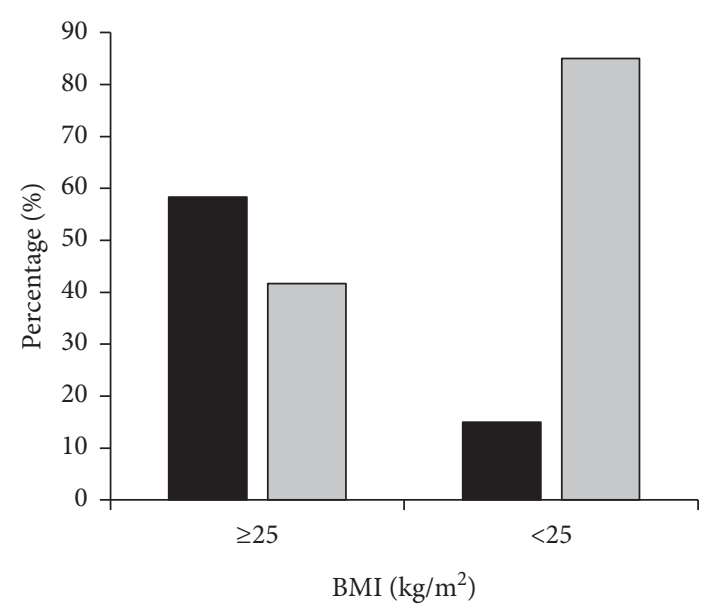

High-risk group

$\square$ Non-high-risk group

(b)

Figure 7: Analysis on the obese ratio of patients in the two groups.

of nonobese patients in the non-high-risk and high-risk group was $85 \%$ and $15 \%$, respectively, indicating that there was a very marked difference $(P<0.01)$.

\subsection{Logistic Regression Analysis of Influencing Factors of} Prostate Cancer. Logistic regression was employed to analyze various factors related to PCa. As shown in Table 2, age, height, and weight were not correlated with the emerging of high-risk PCa $(P>0.05)$, while PSA, BMI $\geq 25 \mathrm{~kg} / \mathrm{m}^{2}$, and $\mathrm{BMI}<25 \mathrm{~kg} / \mathrm{m}^{2}$ had a remarkable correlation with the emerging of high-risk PCa $(P<0.05)$. In addition, further multifactor logistic regression analysis (Table 3 ) showed that PSA and BMI were greatly correlated with the emerging of high-risk PCa $(P<0.05)$.

3.7. Correlation on Gleason Score and Body Mass Index in Patients with Prostate Cancer. Gleason score of patients with PCa was regarded as a continuous categorical variable factor, changes in BMI values of patients with PCa were for statistics, and Pearson correlation analysis was applied for patients' Gleason score and BMI. Figure 8 indicated that there was an enormously positive correlation between BMI and Gleason score of patients with PCa $(r=0.661$ and $P=0.007)$.

\section{Discussion}

Multiple improved operators were introduced to construct a new multioperator ultrasound imaging algorithm on the basis of MV. Besides, the image quality of ultrasound image obtained from MOA was compared with that of other algorithms. The results showed that the horizontal resolution and image contrast of MOA was relatively stable with continuous increase of $M / N$ value in contrast to other algorithms. Thus, it suggested that MOA improved the robustness of MV to increase its stability. Furthermore, the frame rate and speed-up ratio of MOA were more stable than those of other algorithms. When ultrasound image was processed by MOA, the algorithm not only improved the quality of ultrasound images but also realized the real-time performance of ultrasound imaging.

The incidence of PCa in first-tier cities in China was substantially higher than that of rural areas or underdeveloped areas, indicating that the emerging of PCa might be greatly correlated with region and lifestyle [13]. Studies have shown that patients with high BMI had a higher incidence of various common cancers, including $\mathrm{PCa}$ [14]. The results in the study illustrated that there were great differences in BMI and PSA among patients of the high-risk and non-high-risk group $(P<0.05)$. Some studies have found that changes in blood lipid levels (triglycerides and cholesterol) and BMI were associated with the incidence of PCa. Li et al. [15] found that men with $\mathrm{BMI} \geq 35 \mathrm{~kg} / \mathrm{m}^{2}$ had a greatly higher risk of developing PCa than men with $\mathrm{BMI}<25 \mathrm{~kg} / \mathrm{m}^{2}$, which were similar to the results of the study. The proportion of obese patients with PCa was analyzed to find that the proportion of obese patients increased markedly in contrast to the nonobese patients $(P<0.05)$. In addition, the number of obese patients in the high-risk group was dramatically higher than that of the non-high-risk group $(P<0.05)$, and the number of nonobese patients in the non-high-risk group was considerably higher than that in the high-risk group $(P<0.01)$, suggesting that $\mathrm{BMI} \geq 25 \mathrm{~kg} / \mathrm{m}^{2}$ was a potential risk factor for high-risk PCa.

Some studies have pointed out that the accumulation of fat would stimulate the body to produce cytokines such as cell transformation factor and interleukin, so as to trigger prostatic cells to be activated and thus significantly increase the probability of cancerization [16]. Logistic regression results showed that both PSA and BMI had a huge correlation with the emerging of high-risk PCa $(P<0.05)$. Lee et al. [17] believed that obesity was associated with an increased risk of high-grade $\mathrm{PCa}$, and about $50 \%$ of obese 
TABLE 2: Single-factor logistic regression analysis of patients in the two groups.

\begin{tabular}{|c|c|c|c|c|c|}
\hline Factors & $\chi^{2}$ & $I^{2}(\%)$ & OR & $95 \% \mathrm{CI}$ & $P$ value \\
\hline Age (years old) & 3.67 & 19 & 0.785 & $0.713,1.398$ & 0.813 \\
\hline Height & 4.02 & 21 & 1.553 & $0.675,3.642$ & 0.297 \\
\hline Weight & 4.08 & 17 & 1.043 & $0.965,1.076$ & 0.483 \\
\hline PSA & 0.35 & 9 & 1.068 & $1.078,1.136$ & $\leq 0.001^{*}$ \\
\hline $\mathrm{BMI} \geq 25 \mathrm{~kg} / \mathrm{m}^{2}$ & 0.62 & 4 & 1.083 & $0.928,1.162$ & $\leq 0.001^{*}$ \\
\hline $\mathrm{BMI}<25 \mathrm{~kg} / \mathrm{m}^{2}$ & 0.53 & 12 & 2.457 & $1.365,4.349$ & $0.002^{*}$ \\
\hline
\end{tabular}

* expressed the difference was statistical remarkable.

TABLE 3: Multifactor logistic regression analysis of high-risk PSA

\begin{tabular}{lccccc}
\hline Factors & $\chi^{2}$ & $I^{2}(\%)$ & OR & $95 \%$ CI & $P$ value \\
\hline BMI $\left(\mathrm{kg} / \mathrm{m}^{2}\right)$ & 2.86 & 19 & 6.785 & $1.216,31.081$ & 0.028 \\
PSA & 0.45 & 9 & 2.068 & $0.054,0.915$ & 0.045 \\
\hline
\end{tabular}

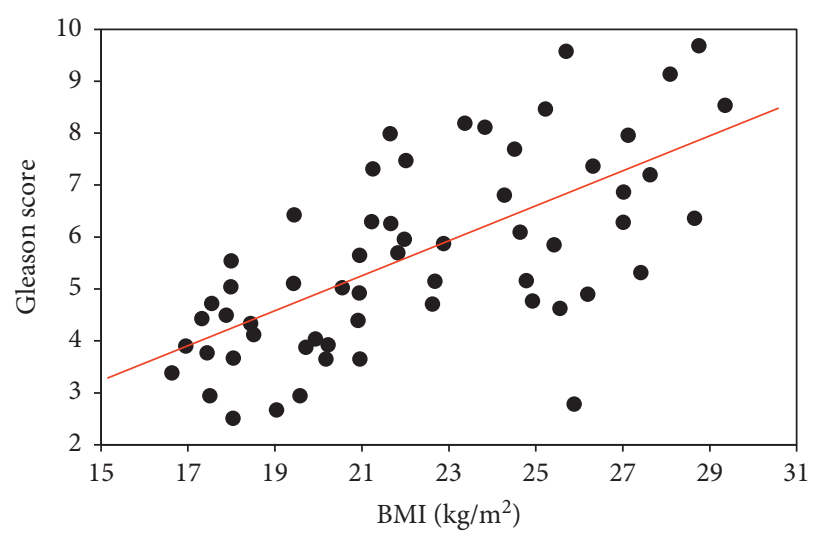

FIgURE 8: Correlation analysis on Gleason score and BMI of patients.

patients would die of PCa within 10 years. Schenk et al. [18] found that abdominal obesity was enormously associated with an increased risk of invasive PCa based on epidemiological data. The correlation analysis of BMI and Gleason score showed a significant positive correlation $(r=0.661$ and $P=0.007)$. Some scholars have found that obesity not only increased the risk of $\mathrm{PCa}$ but also raised Gleason score and mortality of patients [3], which was consistent with results of the study.

\section{Conclusion}

Based on conventional MV, several improved operators were introduced to establish a new multioperator ultrasound imaging algorithm that was applied for the diagnosis of patients with $\mathrm{PCa}$, so as to explore the correlation between $\mathrm{BMI}$ and malignant degree of PCa. However, there were still some deficiencies in the study. The correlation between BMI and PCa was only studied, and there was no analysis on other factors such as triglyceride and cholesterol, which had certain limitations for clinical guidance. In the future, blood lipids and other related factors should be added into the work for analysis to provide major clinical guidance. To sum up, MOA was established in the study based on conventional $\mathrm{MV}$, which could increase the ultrasound image quality and computing speed. BMI was a risk factor for high-risk PCa and was positively correlated with the malignant degree of PCa. This study provided a reference for clinical evaluation of malignant degree of PCa.

\section{Data Availability}

The data used to support the findings of this study are available from the corresponding author upon request.

\section{Conflicts of Interest}

The authors declare no conflicts of interest.

\section{Authors' Contributions}

Yong Wei, Hongru Zhu, and Peng Chen contributed equally to this work.

\section{References}

[1] K. Fujita and N. Nonomura, "Urinary biomarkers of prostate cancer," International Journal of Urology, vol. 25, no. 9, pp. 770-779, 2018.

[2] R. A. Castillejos-Molina and F. B. Gabilondo-Navarro, "Prostate cancer," Salud Pública de México, vol. 58, no. 2, pp. 279-284, 2016. 
[3] K. Wang, X. Chen, T. A. Gerke, V. Y. Bird, H. K. Ghayee, and M. Prosperi, "BMI trajectories and risk of overall and gradespecific prostate cancer: an observational cohort study among men seen for prostatic conditions," Cancer Medicine, vol. 7, no. 10, pp. 5272-5280, 2018.

[4] M. S. Pichardo, C. J. Smith, T. H. Dorsey, C. A. Loffredo, and S. Ambs, "Association of anthropometric measures with prostate cancer among African American men in the nciMaryland prostate cancer case-control study," Cancer Epidemiology Biomarkers \& Prevention, vol. 27, no. 8, pp. 936-944, 2018.

[5] G. Negrão de Figueiredo, K. Müller-Peltzer, V. Schwarze, J. Rübenthaler, and D. Clevert, "Ultrasound and contrast enhanced ultrasound imaging in the diagnosis of acute aortic pathologies," Vasa, vol. 48, no. 1, pp. 17-22, 2019.

[6] S. H. Shin, W. S. Yoo, and H. Choi, "Development of modified RSA algorithm using fixed mersenne prime numbers for medical ultrasound imaging instrumentation," Computer assisted surgery (Abingdon, England), vol. 24, no. 2, pp. 73-78, 2019.

[7] V. Kumar, R. Liu, R. R. Kinnick et al., "Unambiguous identification and visualization of an acoustically active catheter by ultrasound imaging in real time: theory, algorithm, and phantom experiments," IEEE Transactions on Biomedical Engineering, vol. 65, no. 7, pp. 1468-1475, 2018.

[8] K. Song, P. Liu, and D. C. Liu, "Combining autocorrelation signals with delay multiply and sum beamforming algorithm for ultrasound imaging," Medical, \& Biological Engineering \& Computing, vol. 57, no. 12, pp. 2717-2729, 2019.

[9] T. A. Morgan, K. E. Maturen, K. E. Maturen, N. Dahiya, M. R. M. Sun, and A. Kamaya, "US li-rads: ultrasound liver imaging reporting and data system for screening and surveillance of hepatocellular carcinoma," Abdominal Radiology, vol. 43, no. 1, pp. 41-55, 2018.

[10] V. Kumar, M. Katayama, R. Peavler, A. Alizad, M. Belohlavek, and M. Fatemi, "Real-time visualization of an acoustically active injection catheter with ultrasound imaging: algorithm and in vivo validation in a swine model," IEEE Transactions on Biomedical Engineering, vol. 66, no. 11, pp. 3212-3219, 2019.

[11] A. Cafarelli, L. A. Chanel, F. Di Bartolo et al., "Ultrasound acoustic radiation force impulse imaging for high intensity focused ultrasound focal spot localization," Annual International Conference of the IEEE Engineering in Medicine and Biology Society. IEEE Engineering in Medicine and Biology Society. Annual International Conference, vol. 2018, pp. 5713-5716, 2018.

[12] C. J. Kane, S. E. Eggener, A. W. Shindel, and G. L Andriole, "Variability in outcomes for patients with intermediate-risk prostate cancer (Gleason score 7, International Society of Urological Pathology Gleason Group 2-3) and implications for risk stratification: a systematic review," European urology focus, vol. 3, no. 4-5, pp. 487-497, 2017.

[13] N. M. Davies, T. R. Gaunt, T. R. Gaunt et al., "The effects of height and BMI on prostate cancer incidence and mortality: a Mendelian randomization study in 20,848 cases and 20,214 controls from the PRACTICAL consortium," Cancer Causes \& Control, vol. 26, no. 11, pp. 1603-1616, 2015.

[14] N. Bansal, M. Bartucci, S. Yusuff et al., "BMI-1 targeting interferes with patient-derived tumor-initiating cell survival and tumor growth in prostate cancer," Clinical Cancer Research, vol. 22, no. 24, pp. 6176-6191, 2016.

[15] J.-B. Li, S. Luo, M. C. S. Wong et al., "Longitudinal associations between BMI change and the risks of colorectal cancer incidence, cancer-relate and all-cause mortality among 81,388 older adults," BMC Cancer, vol. 19, no. 1, p. 1082, 2019.

[16] L. E. Hackshaw-McGeagh, C. M. Penfold, E. Walsh, and J. Donovan, "Physical activity, alcohol consumption, BMI and smoking status before and after prostate cancer diagnosis in the ProtecT trial: opportunities for lifestyle modification," International Journal of Cancer, vol. 137, no. 6, pp. 1509-1515, 2015.

[17] C.-H. Lee, C. Lin, C.-Y. Wang et al., "Premorbid BMI as a prognostic factor in small-cell lung cancer-a single institute experience," Oncotarget, vol. 9, no. 37, pp. 24642-24652, 2018.

[18] J. M. Schenk, M. L. Neuhouser, S. J. Beatty et al., "Randomized trial evaluating the role of weight loss in overweight and obese men with early stage prostate cancer on active surveillance: rationale and design of the prostate cancer active lifestyle study (PALS)," Contemporary Clinical Trials, vol. 81, pp. 34-39, 2019. 